\title{
Age dependence of metallicity gradients in the Galactic disc from astrometry and asteroseismology
}

\author{
Luca Casagrande \\ Research School of Astronomy \& Astrophysics, Mount Stromlo Observatory \\ The Australian National University, ACT 2611, Australia \\ email: luca.casagrande@anu.edu.au
}

\begin{abstract}
Asteroseismology allows us to determine stellar parameters (distances, masses and ages) independently from Gaia astrometry, and it provides us with a new and complementary tool for studying stellar populations in the Galaxy. The prospects and synergies that asteroseismic and astrometric space-borne missions reserve to the field of Galactic archaeology are marvellous, and results have already started to emerge. For example, the study of metallicity gradients as function of age will provide powerful constraints to understand the evolution of the Milky Way disc at high-redshift.
\end{abstract}

Keywords. Galaxy: stellar content, Galaxy: disc, Galaxy: evolution, stars: fundamental parameters, stars: oscillations, surveys, techniques: photometric

\section{Asteroseismology: the new kid on the block}

Red giant stars are ideal targets to decipher the formation history of the Milky Way: on the HR diagram they span a vastly different range of gravities and luminosities, thus probing a large range of distances. Their ages essentially cover the entire history of the Universe, thus making them fossil remnants from different epochs of the formation of the Galaxy. The cold surface temperatures encountered in red giants are the realm of interesting atomic and molecular physics shaping their emergent spectra. This temperature regime is also dominated by convection, which is the main driver of the oscillation modes that we are now able to detect in several thousands of stars thanks to space borne asteroseismic missions such as CoRoT, Kepler/K2, and soon to be TESS. By measuring oscillation frequencies in stars, asteroseismology allows us to measure fundamental physical quantities, masses and radii in particular, which otherwise would be inaccessible in single field stars. Masses and radii can then be used to obtain information on stellar distances and ages (e.g., Hekker \& Christensen-Dalsgaard 2016, for a recent review).

Asteroseismic ages are independent from astrometric distances (although the two methods can, and should, be combined to place tighter observational constraints), and thus provide a new and powerful tool for all photometric and spectroscopic stellar surveys. Even when accurate astrometric distances are available to allow comparison of stars with isochrones, the derived ages are still uncertain, and statistical techniques are required to avoid biases. Furthermore, isochrone dating is meaningful only for stars in the turnoff and subgiant phase, where stars of different ages are clearly separated in the HR diagram. This is in contrast, for example, to stars on the red giant branch, where isochrones with vastly different ages can fit equally well observational constraints such as effective temperatures, metallicities and surface gravities within their errors (e.g., Soderblom 2010, for a review). Thus, asteroseismology is the only way forward to determine ages of red giant stars. 

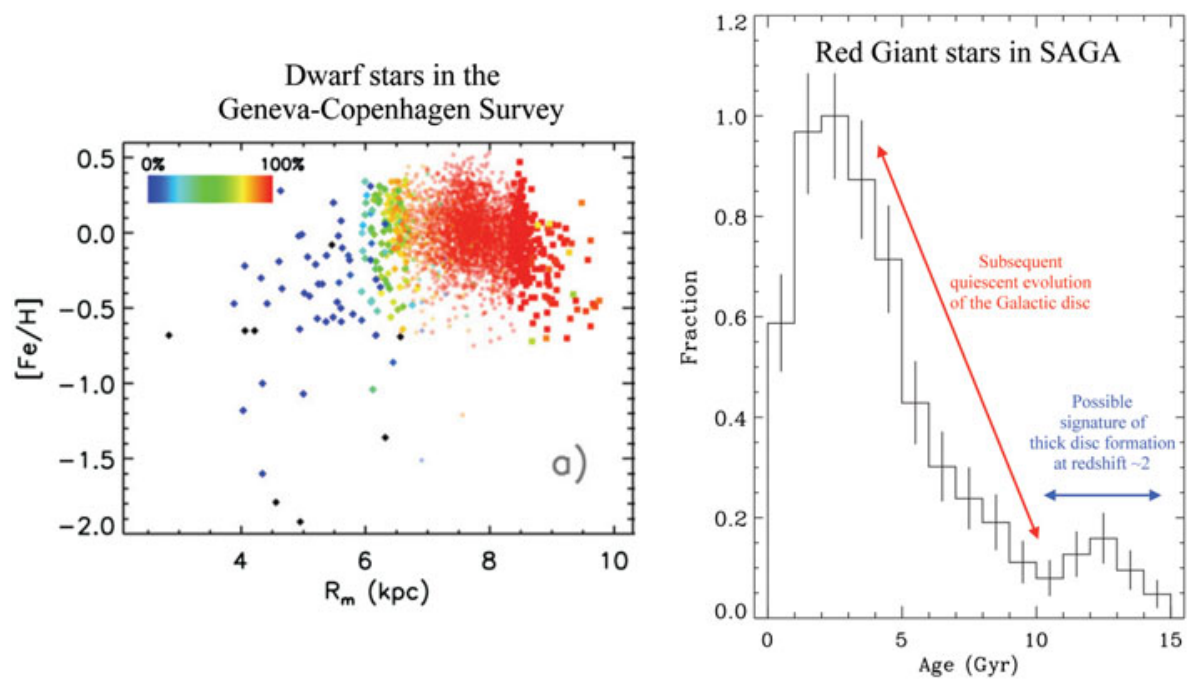

Figure 1. Left panel: metallicities of stars in the Geneva-Copenhagen Survey as function of their orbital radii. The probability of a star to belong to the thin disc is represented by colour (adapted from Casagrande et al. 2011). Right panel: age distribution of red giant stars in the Kepler field and SAGA (adapted from Casagrande et al. 2016).

\section{Vertical \& radial gradients from asteroseismology and astrometry}

In Casagrande et al. (2011) we have exploited the power of combining Strömgren photometry with Hipparcos astrometry for the sake of studying stellar populations in the Solar Neighbourhood. We found that the radial metallicity gradient changes as function of time. Thick disc stars are preferentially older, and with a positive radial metallicity gradient (diamonds in Figure 1, left panel. See also discussion of Figure 18 in Casagrande et al. 2011). This positive metallicity gradient for thick disc stars might hold clues on the formation of the Milky Way thick disc at high-redshift (Kawata et al. 2017).

As part of the Strömgren survey for Asteroseismology and Galactic Archaeology (SAGA, Casagrande et al. 2014), we have then combined Strömgren photometry with Kepler asteroseismology. A direct measurement of the vertical age gradient of the Milky Way disc returns approximately $4 \mathrm{Gyr} / \mathrm{kpc}$, though characterised by a large dispersion of ages at all heights. Once target selection effects are taken into account (e.g., Casagrande 2016), the smooth age distribution of red giant stars over the last 10 Gyr is consistent with that of a stellar population having a constant star formation rate; this is indicative of a rather quiescent evolution for the Milky Way disc since a redshift $z \simeq 2$ (modulo shifts on the absolute scale of stellar ages). More interestingly, the bump in the distribution at older ages could be indicative of thick disc formation (Figure 1, right panel. See Casagrande et al. 2016, for a more detailed discussion).

\section{References}

Casagrande, L., Schönrich, R., Asplund, M., et al. 2011, A $\xi A$, 530, A138

Casagrande, L., Silva Aguirre, V., Stello, D., et al. 2014, ApJ, 787, 110

Casagrande, L., Silva Aguirre, V., Schlesinger, K. J., et al. 2016, MNRAS, 455, 987

Casagrande, L. 2016, Astronomische Nachrichten, 337, 889

Hekker, S. \& Christensen-Dalsgaard, J. 2016, arXiv:1609.07487

Kawata, D., Allende Prieto, C., Brook, C. B., et al. 2017, MNRAS, submitted.

Soderblom, D. R. 2010, ARAESA, 48, 581 